\title{
What dictates the position of the diaphragm-The heart or the liver?
}

\author{
A review of sixty-five cases
}

\begin{abstract}
Case histories of 65 patients with cardiac malposition were reviewed to assess abdominal taxis and hemidiaphragm posture. We present their roentographic findings to support the hypothesis that the cardiac mass determines the caudad displacement and lower position of the related hemidiaphragm. The popular hypothesis that the liver lifts the corresponding hemidiaphragm is questioned. Incidentally, a review of their echocardiographic findings demonstrate the presence of complex congenital heart defects in a majority of these patients. (J THORAC CardiovasC Surg 1994;108:687-91)
\end{abstract}

Veerappa Reddy, MB, MS, MCh, Sanjeev Sharma, MD, and Adnan Cobanoglu, MD, Portland, Ore.

I normal individuals, that is, a population with situs solitus and levocardia, the left hemidiaphragm is always at a lower position than the right hemidiaphragm (Fig. 1). This is a constant observation relevant to all ages. A widely believed hypothesis is that the liver is responsible for the higher position of the right hemidiaphragm. This misconception has been frequently described in the medical literature and has been taught to medical students and residents. Our review of children with cardiac malposition with or without abdominal heterotaxy led us to question the validity of this popular hypothesis.

\section{Patients and methods}

Since 1979, 65 infants with cardiac malposition with a variety of congenital intracardiac defects were referred to the Division of Cardiopulmonary Surgery, Oregon Health Sciences University. Of the 65 infants, $46(71 \%)$ had isolated dextrocardia, $8(12 \%)$ had dextroversion, $6(9 \%)$ had mesocardia, and 5 $(8 \%)$ had levoversion. These patients charts, roentograms, and echocardiograms were reviewed to determine thoracic and

From the Division of Cardiopulmonary Surgery, Oregon Health Sciences University, Portland, Ore.

Received for publication Jan. 27, 1994.

Accepted for publication May 31, 1994.

Address for reprints: Adnan Cobanoglu, MD, Professor and Chairman, Division of Cardiopulmonary Surgery, Oregon Health Sciences University, L323, 3181 SW Sam Jackson Park Rd., Portiand, OR 97201.

Copyright ${ }^{\circledR} 1994$ by Mosby-Year Book, Inc.

$0022-5223 / 94 \$ 3.00+0 \quad \mathbf{1 2} / \mathbf{1} / \mathbf{5 8 2 0 0}$ abdominal situs and their influence on the position of the diaphragm.

Thoracic and abdominal situs was determined by the position of the cardiac apex, stomach, and the major lobe of the liver. Accordingly, these patients were classified as having isolated dextrocardia, dextroversion, levoversion, or mesocardia. Isolated dextrocardia is defined as a right-sided cardiac apex with abdominal situs solitus. Dextroversion is a dextrocardia with abdominal situs inversus. Levoversion is levocardia with abdominal situs inversus. Mesocardia is a midline heart with an undetermined cardiac apex and abdominal situs ambiguous. Those patients with mediastinal shift and cardiac position changes caused by other disease processes were excluded from our discussion. Further, a detailed review of the abdominal heterotaxy is beyond the scope of this paper.

Transthoracic and, more recently, transesophageal echocardiograms were reviewed to determine the occurrence of cardiac defects. To determine the effect of ventricular force on the position of the diaphragm, we classified the hearts as having single or double ventricle.

\section{Findings}

Of the patients with isolated dextrocardia, 25 (54\%) were male and $21(46 \%)$ were female. Fifteen infants $(33 \%)$ had normal hearts, whereas 31 patients $(67 \%)$ had complex cardiac anomalies (Table I). In patients with isolated dextrocardia on supine posteroanterior chest roentgenograms, the cardiac apex was in the right side of the chest, the major lobe of the liver was on the right side, and the gastric bubble was on the left side. In all patients, the right hemidiaphragm was noted to be significantly lower than the left hemidiaphragm (Fig. 2).

Of the eight patients with dextroversion, six (75\%) were male and two $(25 \%)$ were female. All patients had 


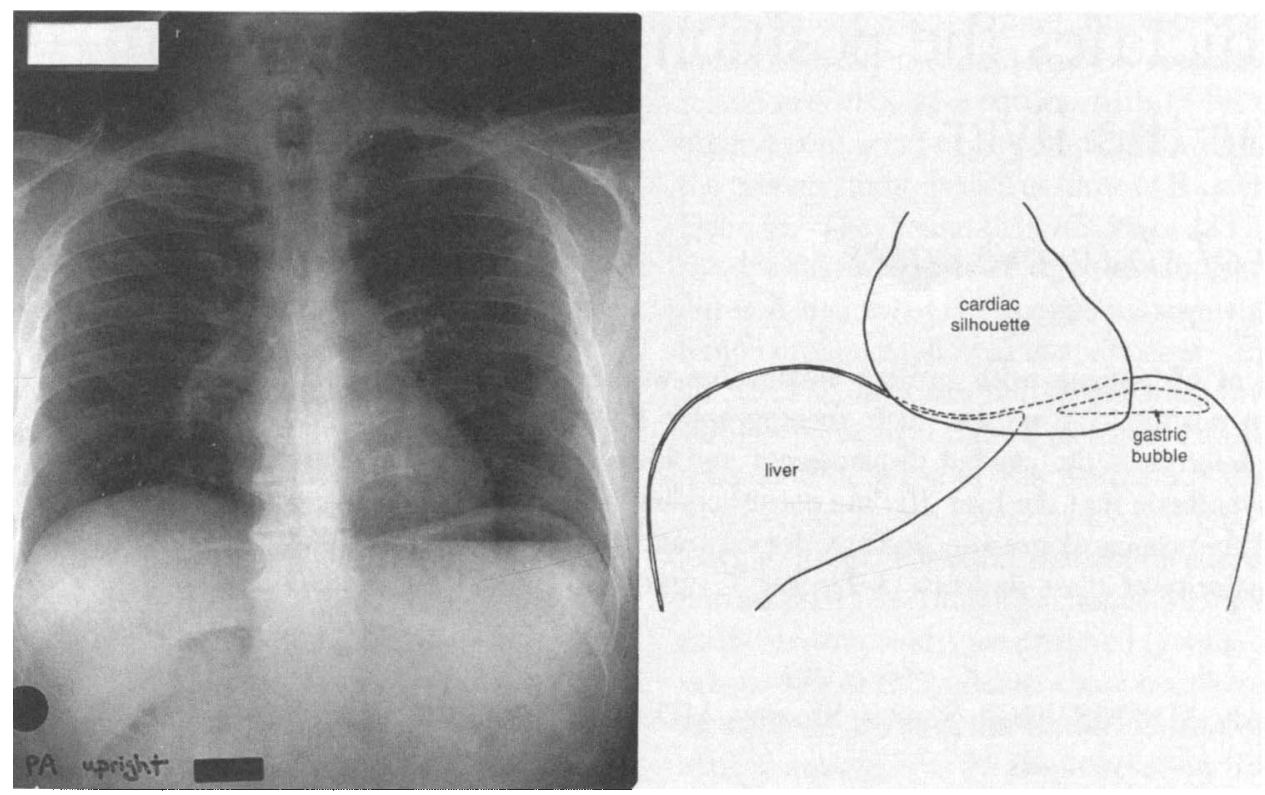

Fig. 1. Normal posture of hemidiaphragms and abdominal situs solitus.

Table I. Relationship of cardiac anomalies with respect to cardiac malposition

\begin{tabular}{|c|c|c|c|c|}
\hline $\begin{array}{l}\text { Cardiac anomaly } \\
(\mathrm{n}=50)\end{array}$ & $\begin{array}{l}\text { Isolated dextrocardia } \\
\qquad(\mathrm{n}=46)\end{array}$ & $\begin{array}{l}\text { Dextroversion } \\
\quad(\mathrm{n}=8)\end{array}$ & $\begin{array}{l}\text { Levoversion } \\
\quad(\mathrm{n}=5)\end{array}$ & $\begin{array}{l}\text { Mesocardia } \\
\quad(\mathrm{n}=6)\end{array}$ \\
\hline Single ventricle & 7 & 2 & 1 & 1 \\
\hline Complex TGA & 7 & 5 & & \\
\hline TOF & 4 & & 3 & 2 \\
\hline HLHS & 4 & 1 & & . \\
\hline CAVC & 3 & & 1 & 1 \\
\hline PA-IVS & 2 & & & \\
\hline Scimitar syndrome & 1 & & & 2 \\
\hline ASD & 1 & & & \\
\hline PASVC & 1 & & & \\
\hline Vent inv, VSD & 1 & & & \\
\hline
\end{tabular}

$T G A$, Transposition of great arteries; TOF, tetralogy of Fallot; $H L H S$, hypoplastic left heart syndrome; CAVC, complete atrioventricular canal defect; $P A-I V S$, pulmonary atresia with intact ventricular septum; $A S D$, atrial septal defect; $P A S V C$, partial anomalous systemic venous connection; $V e n t$ inv, ventricular inversion; $V S D$, ventricular septal defect.

complex cardiac defects (see Table I). In these patients, on supine posteroanterior chest roentgenograms, the cardiac apex was in the right side of the chest, the major lobe of the liver was on the left side, and the gastric bubble was on the right side. The right hemidiaphragm was always noted to be at a significantly lower level than the left hemidiaphragm (Fig. 3).

Three boys (60\%) and two girls (40\%) comprised those patients with levoversion. All had complex cardiac defects (Table I). On supine posteroanterior chest roentograms, the cardiac apex was in the left side of the chest, the major lobe of the liver was on the left side, and the gastric bubble was on the right side. The left hemidiaphragm was significantly lower than the right hemidiaphragm in all of these patients (Fig. 4).

In those six patients with mesocardia, three (50\%) were boys and three $(50 \%)$ were girls. All patients had complex cardiac defects (see Table I). On supine posteroanterior chest roentgenograms, the cardiac apex was in the midline, and the gastric and liver situs was ambiguous. Accordingly, both hemidiaphragms were at the same level (Fig. 5).

Of these 65 patients, $50(77 \%)$ had complex cardiac defects and $15(23 \%)$ had normal hearts. Nineteen patients (38\%) had single ventricle and $31(62 \%)$ had double ventricle. 

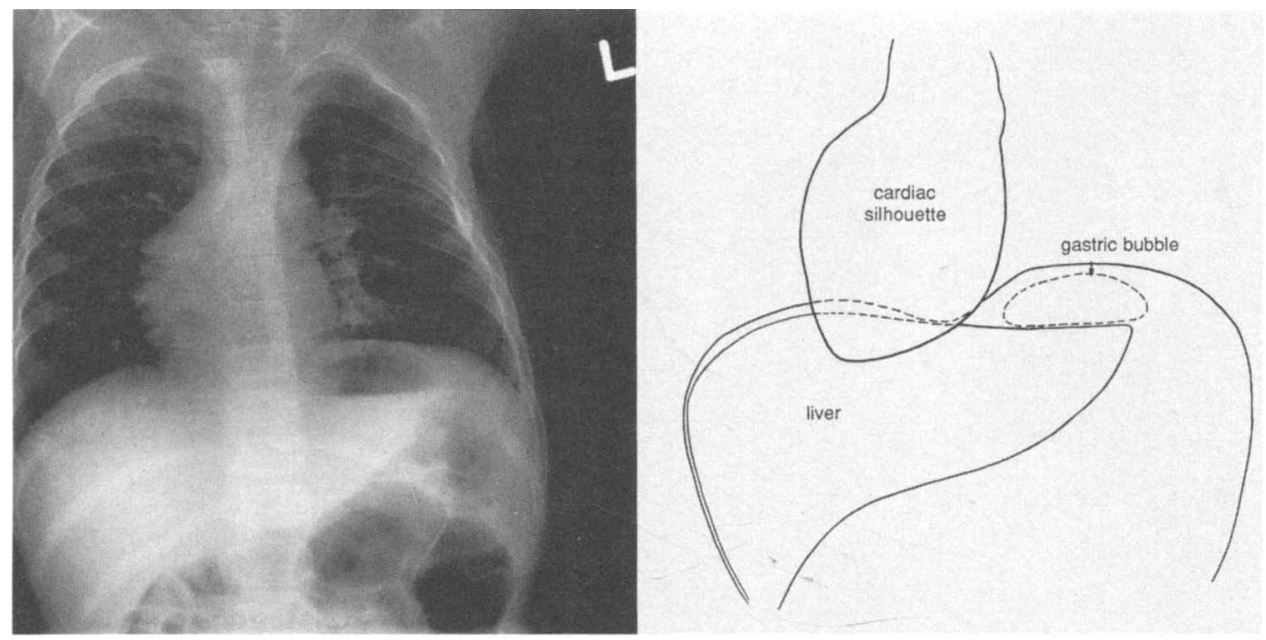

Fig. 2. Isolated dextrocardia: cardiac apex to the right, right hemidiaphragm at lower position, and abdominal situs solitus.
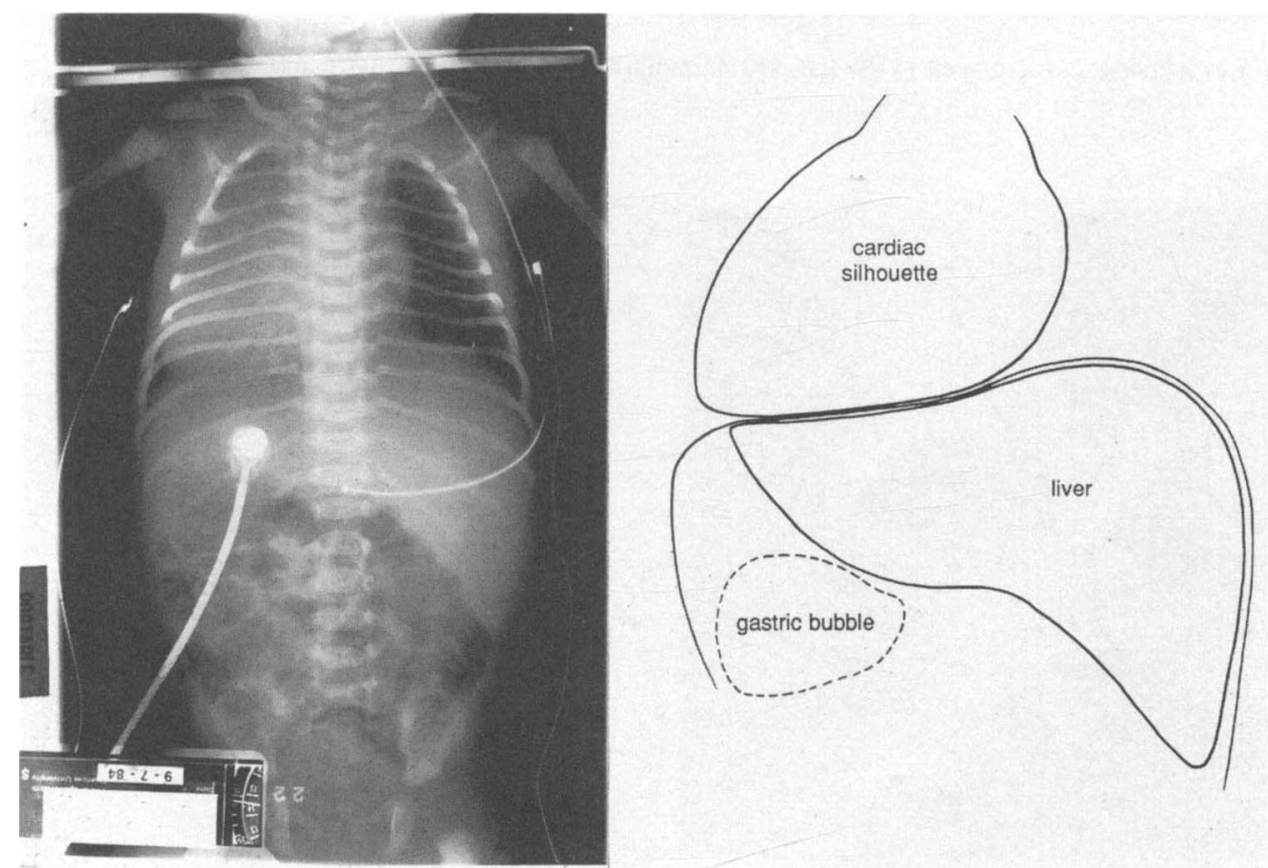

Fig. 3. Dextroversion: cardiac a pex to the right, right hemidiaphragm at lower position, and abdominal situs inversus.

\section{Discussion}

Anatomists in the 1920 s and 1930 s attempted to explain that hemidiaphragm position is determined by the cardiac mass, but with minimal evidence to support their hypotheses. ${ }^{1}$ Again, in the 1960s, the cardiac hypothesis was professed by a few physicians who had strong roentgenographic evidence for their claim. ${ }^{2,3}$ However, they received little attention from the medical community. Over the past three decades, the classic teaching has been that the diaphragm is elevated in the right side because the liver is in the right side. Our group thought that it is timely to address this issue again and to elucidate the fact that it is the cardiac mass and not the liver that determines the posture of the hemidiaphragms. 

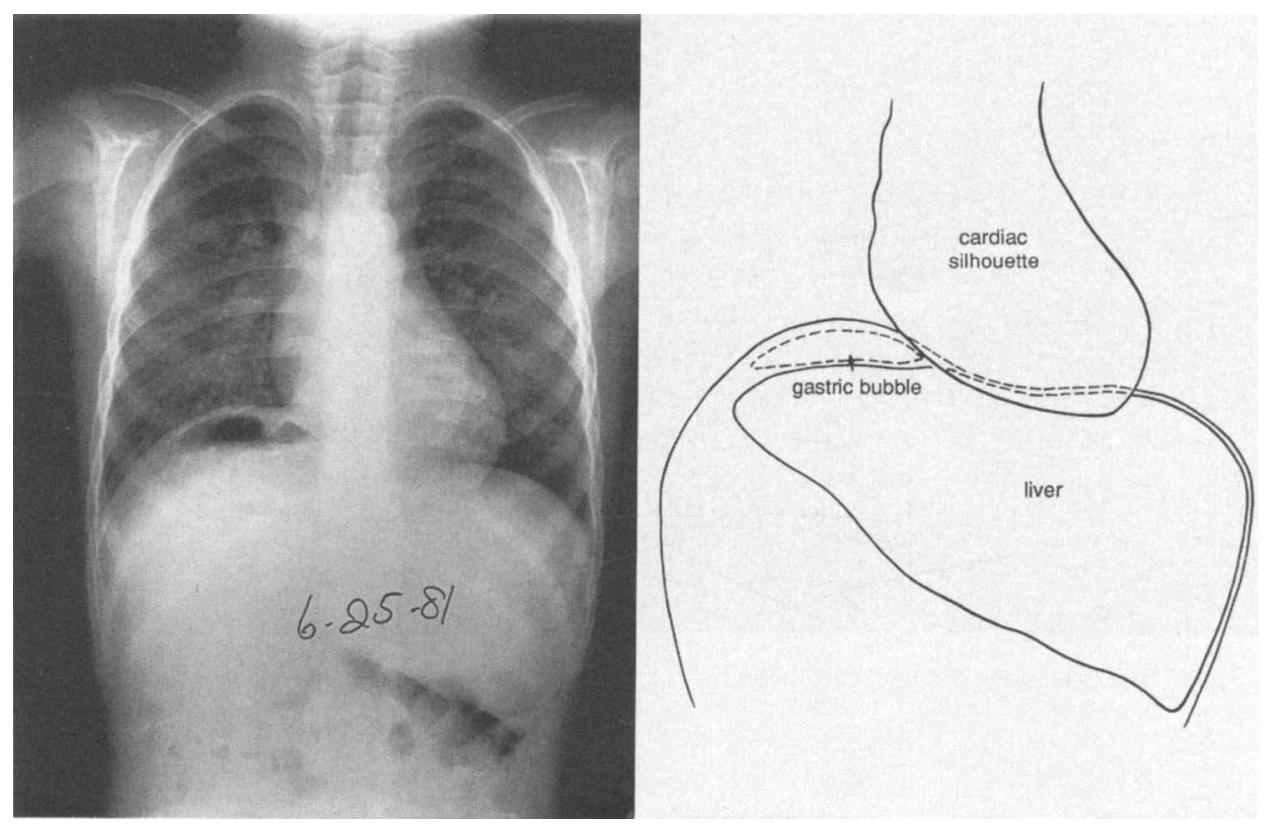

Fig. 4. Levoversion: cardiac apex to the left, left hemidiaphragm at lower position, and abdominal situs inversus.

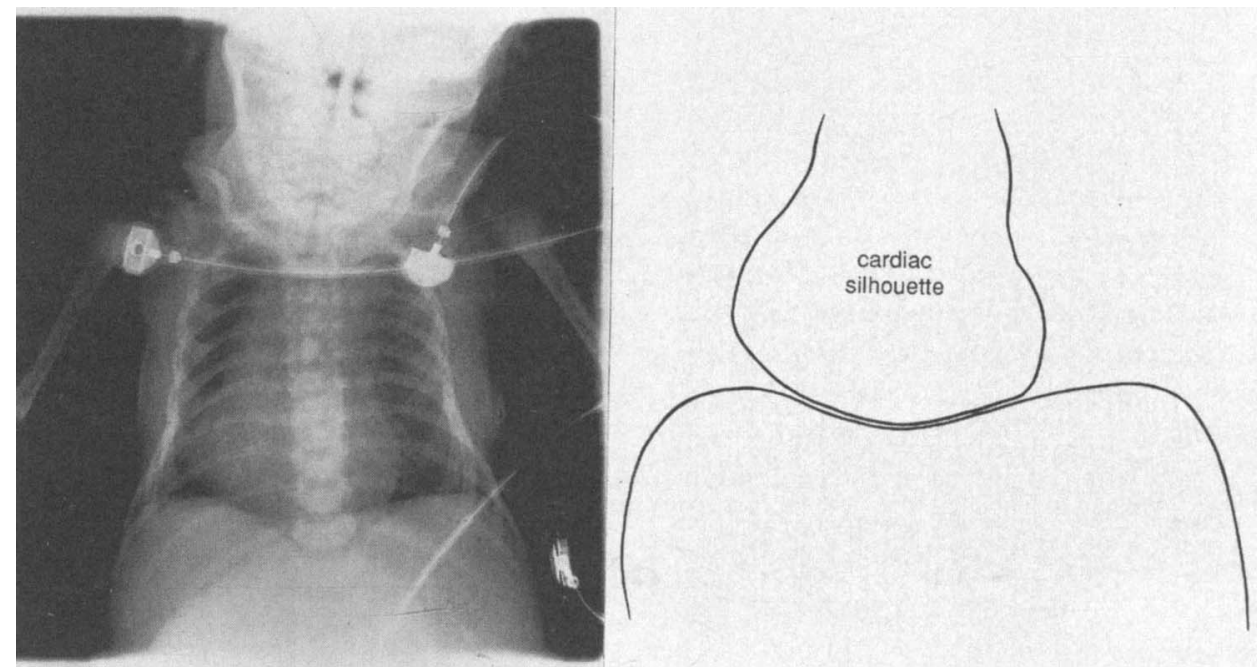

Fig. 5. Mesocardia: ambiguous cardiac apex and abdominal situs, and hemidiaphragms at equal position.

Our group of 65 patients demonstrated that the cardiac mass is what determines the relatively lower position of either diaphragm. In those patients with isolated dextrocardia, although the major lobe of the liver was on the right side, the right hemidiaphragm was at an appreciably lower level than the left hemidiaphragm. Those patients with dextroversion had a right cardiac apex and a left-sided liver; correspondingly, the right hemidia- phragm was at a lower level than the left. In levoversion, even though the cardiac apex and the major lobe of the liver were both on the left side, the left hemidiaphragm was significantly at a lower position. In those patients with an ambiguous cardiac apex and abdominal taxis (i.e., mesocardia), the hemidiaphragms were even in position. Further, irrespective of ventricular physiology, that is, either a single or double ventricle, the determinant was the 
position of the cardiac apex. Also, ventricular physiology did not change the depth of disparity in hemidiaphragm posture.

Incidentally, the majority of patients in our groups with malposition had complex congenital heart defects. Normal hearts $(33 \%)$ were present only in those patients with isolated dextrocardia. Those patients who had dextroversion, levoversion, or mesocardia had relatively more primitive hearts (see Table I).

In summary, it is the cardiac mass that is responsible for the lower position of the corresponding hemidiaphragm. The popular hypothesis that the liver lifts its related hemidiaphragm is false. Further, an unusual hemidiaphragm position should alert the astute physician to the potential presence of cardiac malposition and intracardiac defects. We believe that a more detailed cardiac workup is necessary in these patients.

\section{REFERENCES}

1. Lichtman SS. Isolated congenital dextrocardia. Arch Int Med 1931;48:683-717.

2. Shaher RM, Johnson AM. Isolated laevocardia and isolated dextrocardia: pathology and pathogenesis. Guys Hosp Rep 1963;112:127-51.

3. McGaff CJ, Williams JB, Leight L, Little JA. The rightsided heart: a report of forty-seven cases. Arch Int Med 1963;111:483-7. 